\title{
A RARE COMPLICATION OF ACUTE COLECISTITIS
}

\author{
M. Coțofană ${ }^{1}$, M.B. Șerban ${ }^{1,2}$, D. Ion ${ }^{1,2}$, Adriana Elena Nica ${ }^{2,3}$, Florentina Mușat ${ }^{2}$, Ioana \\ Coțofană ${ }^{4}$, D.N. Păduraru, ${ }^{1,2}$ \\ ${ }^{1}$ III'rd Department of General Surgery, University Emergency Hospital Bucharest, Romania \\ ${ }^{2}$ The University of Medicine and Pharmacy "Carol Davila" Bucharest, Romania \\ ${ }^{3}$ Department of Anaesthesiology, University Emergency Hospital Bucharest, Romania \\ 4“C.I. Parhon” National Institute of Endocrinology
}

Corresponding author: Dan Nicolae Păduraru

Phone no. 0040744756443

E-mail: dan.paduraru.nicolae@gmail.com

\begin{abstract}
Laparoscopic procedures are often preferred to open surgery due to their many advantages, they may sometimes cause rare complications like intraabdominal, pelvic, and abdominal wall abscesses. We report a case of a 45-year-old woman who presented on multiple occasions to the hospital with symptoms suggesting an infection involving the epigastric area. Following an exploratory procedure it was concluded that unretrieved gallstones from a previous laparoscopic cholecystectomy caused an abdominal wall abscess. This case shows that when establishing a diagnosis, the physician should consider that laparoscopic procedures can cause rare complications even after a long period of time.
\end{abstract}

Keywords: laparoscopic cholecystectomy, abdominal wall abscess, gallstones

\section{Introduction}

The popularity of laparoscopic cholecystectomy is mainly because of the advantages that it offers to the patient, like smaller surgical scars, less pain following surgery, shorter hospital stay, faster return to normal activity, reduced risk of infection. However, the perforation of the gallbladder, a frequent event which occurs during this routine procedure and reported in studies of up to $40 \%$ of cases [1], may rarely lead to complications like intraabdominal, pelvic, and abdominal wall abscesses. The main cause of this rare complications is the spillage of gallstones which in most cases is a benign event without serious consequences. In other words, a procedure supposed to be minimally invasive can become one with not only an increased cost but also with a greater discomfort for the patient. Complications that result from these stones are said to occur in $0.08 \%-0.3 \%$ of patients [2].The aim of this case report is to remind that unretreived gallstones from a laparoscopic cholecystectomy can be an important source of late complications involving the peritoneal cavity or the abdominal wall.

\section{Case report}

A 45-year-old woman with symptomatic gallstone disease underwent a laparoscopic cholecystectomy in May 2015. During the procedure, spillage of calculi occurred in the peritoneal cavity. The attempt to remove the 
calculi lead to stone crumbling and spillage of the fragments into the abdominal wall near the epigastric port. The epigastric incision was irrigated and closed with an absorbable suture (Figure 1).

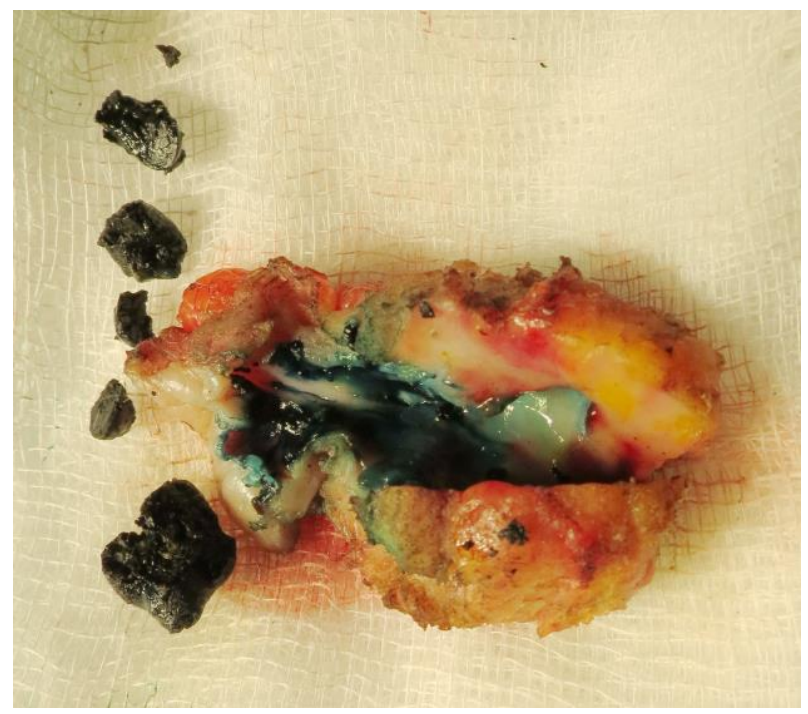

Figure 1 - Gallstone

8 days postoperatively, the patient developed a wound infection at this incision site which was opened and drained. The wound failed to heal and the infection relapsed multiple times, over a period of 7 months, accompanied by pain, redness and tenderness involving the area circumscribing the epigastric port. Last time the patient presented to hospital for this symptomatology, she was admitted for further investigations. The patient had a normal white blood cells count of 6 . Samples from the wound were tested for pathogens and found positive for Klebsiella spp. An ultrasound of the abdominal cavity did not reveal any evidence of fluid accumulation or other collection in the peritoneal cavity. By this time, it was decided that the wound must be explored under local anesthesia. Exploration revealed a long sinus tract which extended down to the rectus abdominis muscles plan where it formed a cavity. 4 black gallstones, with different dimensions (the biggest stone diameter was approximately $6 \mathrm{~mm}$ ), were retrieved from the abscess cavity. The dissection reached down to the level of the round ligament and the sinus tract was excised.The patient was discharged 2 days later having prescribed oral antibiotics for 1 week.

\section{Discussions}

There are some factors that influence the incidence of gallstones spillage during a laparoscopic cholecystectomy (LC):

1. The LC involves maneuvers of grasping the gallbladder with forceps, dissecting it off the liver bed and extracting it through the port site. All these procedures can damage and perforate the gallbladder wall. One can consider replacing the potentially damaging graspers with a new gentler instrument, while at the same time obtaining the adequate traction [3]. A way to facilitate dissection is to aspirate the gall bladder full of bile in order to ease the tension on the wall.

2. In acute inflammation, the gallbladder wall becomes edematous, friable, or gangrenous, increasing the risk of perforation.

3. The chance of a stone spillage event occurring after a gallbladder perforation depends greatly on the size of the perforation, number and size of the gallstones, and the surgeon's expertise.

\section{Gallbladder perforation sollutions}

If a perforation of the gallbladder wall occurs during the surgery contamination can be managed by the use of suction or by applying a grasper, ligature or a surgical clip. In the case of a stone spillage event, all the accessible stones must be retrieved and kept out of inaccessible areas of the peritoneal cavity. In the majority of cases, spilled stones can be completely retrieved. In order to reduce bacterial contamination, abundant irrigation of the peritoneal cavity should be applied. Large amounts of saline facilitate stone retrieval by providing a fluid-gas interface in which hydrophobic gallstones often float. Extra ports, 30-degree scope, liver retractor, and shuttle stone collectorare also helpful in retrieving the lost stones. It would be helpful for early diagnosis of possible complications that the patient's medical records mention if a stone spillage event occurred during surgery. If the patient is also informed about these events, a detailed anamnesis can be very useful in clinical diagnosis.

Abscess formation and associated inflammation can develop as complications after 
surgical intervention in an infectious cholecystitis. They can manifest between several days to as long as 20 years after the surgery with a peak incidence usually around 4 months.4In the mentioned case the patient first presented with symptoms suggesting possible postoperative complications 8 days after surgery. The body's ability to fight infection depends on the efficiency of the immune system reaction, which can be reduced in elderly patients. In many cases patients presenting with an abscess can be afebrile and have a normal white cell count as in this case study.

There are few reported cases in which the nature of the retrieved stones is mentioned. However, this could prove to be a useful information in light of a study which implies that pigment stones are more likely to precipitate an abscess than cholesterol stones. Stewart et al. [5] examined 32 pigment stones and 35 cholesterol stones and found that $78 \%$ of the pigment stones had evidence of bacterial microcolonies whereas none of the cholesterol stones showed evidence of bacteria. In our case black stones wereretrieved, which may indicate they contain high concentrations of pigments.

\section{Diagnostic methods}

In the presented case, diagnosis of abdominal wall abscess was established based on physical examinationand anamnesis which revealed a recent laparoscopic. The diagnosis was later confirmed by exploratory surgery. Still, radiological investigations can sometimes be useful for establishing a diagnosis of such complications especially when they occur after a long period of time post-cholecystectomy. Also, an ultrasound scan can show hyperechoic images with posterior acoustic shadowing that roll over with percutaneous ultrasound-guided injection of normal saline into the abscess cavity. However, radiological investigations sometimes lack in sensitivity as was demonstrated in a study in which spilled stones were identified in $9 \%$ of patients with retained intraabdominal gallstones [6]. CT investigation can reveal spilled stones as hyperdense lesions, but often misses stones with low calcium content which can be identified by ultrasound [7]. MRI technique is able to detect intraabdominal stones as foci of low-signal intensity [8], but it is not sensible enough to detect stones embedded in abscesses when compared with CT scan (it is difficult to differentiate between stones and gas in an abscess on MRI). However, a nonopaque calculus within an abscess may not be visualized by any of the standard imaging techniques and could result in confusion with the diagnosis of abscess due to other causes like actinomycosis or tumor.

\section{Conclusions}

In the case of a stone spillage event, it is not recommended to perform an open laparotomy in order to retrieve any lost stones because they rarely cause complications. However, reasonable effort should be made in order to retrieve an escaped gallstone. Medical records should mention any gall bladder perforation during a LC and also the patient should be informed about it so that if complications arise after a long period of time, they should be more easily diagnosed.

\section{References}

[1]Vincent Chong and Rishi Ram. Laparoscopic drainage of abdominal wall abscess

from spilled stones post-cholecystectomy. Journal of Surgical Case Reports, 2015, 1-2

[2]T Sathesh-Kumar, A P Saklani, R Vinayagam, R L Blackett. Spilled gallstones during laparoscopic cholecystectomy: a review of the literature. Postgrad Med J 2004;80:77-79. doi: 10.1136/pmj.2003.006023

[3]Arishi AR, Rabie ME, Khan MS, et al. Spilled gallstones: the source of an enigma. JSLS. 2008;12(3):321-325

[4]Rothlin MA, Schob O, Schlumpf R, et al. Stones spilled during cholecystectomy: a longterm liability for the patient. SurgLaparoscEndosc. 1997;7:432-4.

[5]Stewart L, Smith AL, Pellegrini CA, Motson RW, Way LW. Pigment gallstones form as a composite of bacterial microcolonies and pigment solids. Ann Surg. 1987;206:242-250

[6]Manukyan MN, Demirkalem P, Gulluoglu $\mathrm{BM}$, et al. Retained abdominal gallstones during laparoscopic cholecystectomy. Am J Surg. 2005; 189:450-452 
[7]Viera FT, Armellini E, Rosa L, et al. complications arising from dropped gallstones Abdominal spilled stones: ultrasound findings. in laparoscopic cholecystectomy p-patients. Abdom Imaging. 2006;31:564-567 AJR. 2000;174:1441-1445.

[8]Morrin MM, Kruskal JB, Hochman MG,

Saldinger PF, Kane RA. Radiologic features of 